Original Research Paper

\title{
Common Fixed Points of Generalized Cyclic $C$ Class $\psi-\phi-\Lambda$ Weak Nonexpansive Mappings
}

\author{
Sahar Mohamed Ali Abou Bakr \\ Department of Mathematics, Faculty of Science, Ain Shams University, Cairo, Egypt
}

Article history

Received: 22-05-2018

Revised: $18-02-2020$

Accepted: 06-03-2020

Email: saharm_ali@yahoo.com

Saharm_ali@sci.asu.edu.eg
Abstract: This paper shows that if $S$ and $T$ are two joint generalized cyclic $F-\psi-\phi-\Lambda$ weak nonexpansive type mappings, then they have only one common fixed point. In particular, every generalized cyclic $C$ class $\psi-\phi-\Lambda$ weak nonexpansive mapping has a unique fixed point. Hence it extends the results of the attached references of this paper.

Keywords: Fixed Point Theorems, $a b c$ Generalized Contractions and Nonexpansive Mappings, Cyclic Weak $\phi$ and Weak $\psi-\phi$ Contraction and Nonexpansive Mappings

\section{Introduction and Preliminaries}

Since 1922 till now many generalizations of Banach contraction principle (Banach, 1922) have been achieved. For cyclic $\psi-\phi$ mappings, we refer to the references below.

In particulr; Sahar Mohamed Ali Abou Bakr (2013) proved the existence of only one fixed point for both $\{a$, $b, c\}$-ntype and $\{a, b, c\}$-ctype types of mappings defined on closed convex weakly Cauchy subset $C$ of a normed space $X$.

\section{Definition 1}

Let $C$ be a subset of a normed space $X$ and $T$ be a mapping from $C$ into $C$ satisfying:

$$
\begin{aligned}
& \|T(x)-T(y)\| \leq a\|x-y\|+b\|x-T(x)\| \\
& +c \max \{\|y-T(y)\|,\|y-T(x)\|\} \forall x, y \in C, a, b, c \in[0,1] .
\end{aligned}
$$

Then:

(1) $T$ is said to be $\{a, b, c\}$-ntype mapping, if $0<a<1,0$ $<b, 0 \leq c<1=2$ and $a+b+c=1$

(2) $T$ is said to be $\{a, b, c\}$-ctype mapping, if $0 \leq c<1 / 2$ and $a+b+c<1$

Sahar Mohamed Ali Abou Bakr and Ansari (2017) introduced new $\mathfrak{U}-T$ cyclic weak contraction $C$-class concept. Namely; $\mathfrak{U}-T$ cyclic weak $F-\psi$ - $\phi$-contraction type and proved some related fixed point theorems.

\section{Definition 2}

Let $S$ and $T$ be self mappings on $X$. Then $S$ is $\mathfrak{U}-T$ cyclic $F-\psi-\phi$ weak contraction mapping on $X$ iff there are:
(1) A collection of non empty sets $\mathfrak{U}=\left\{A_{i}\right\}_{i=1}^{j}$ with $X=\bigcup_{i=1}^{j} A_{i}$

(2) Non-decreasing functions $\psi, \phi:[0, \infty] \rightarrow \mathbb{R}^{+}, \psi(t)=0$ iff $t=0$ and $\phi(t)=0$ iff $t=0$ with $\psi$ continuous, and

(3) A $C$ class function $F$ : That is; $F$ : $[0, \infty] \times[0, \infty] \rightarrow \mathbb{R}$ is continuous and satisfying $F(u, v) \leq u$ for all $u$, $v \in[0, \infty]$ and if $F(u, v)=u$, then either $u=0$ or $v=0$ such that:

(1) $\mathfrak{U}$ is a $T$-cyclic representation of $X$ with respect to $S$ : That is; $T\left(S\left(A_{1}\right)\right) \subset A_{2}, T\left(S\left(\left(A_{2}\right)\right)\right) \subset A_{3}, \ldots, T\left(S\left(A_{j-1}\right)\right) \subset$ $A_{j}$ and $T\left(S\left(\left(A_{j}\right)\right)\right) \subset A_{1}$

(2) The following contractivity condition is satisfied:

$$
\begin{aligned}
& \psi(d(T(S(x)), T(S(y)))) \\
& \leq F(\psi(d(T(x), T(y))), \phi(d(T(x), T(y))))
\end{aligned}
$$

for every $x \in A_{i}, y \in A_{i+1}, i=1,2, \ldots, j$, where $A_{j+1}=A_{1}$.

In this study; we define the real valued function $\Lambda_{S,(a b c):}: X \times X \rightarrow R^{+}$as follows:

$$
\begin{aligned}
& \Lambda_{S,(a b c)}(x, y)=a d(x, y)+b d(x, S(x)) \\
& +c \max \{d(y, S(y)), d(y, S(x))\} \\
& \forall x, y \in X,
\end{aligned}
$$

where, $a, b, c$ are three real numbers.

\section{Definition 3}

Let $(X, d)$ be metric space with $X=A \cup B$ and $S$ be a self mapping on $X$ with: 
(1) $S(A) \subset B$ and $S(B) \subset A$ and

(2) There are real constants $a, b, c \in[0,1]$ with:

$$
d(S(x), S(y)) \leq \Lambda_{S,(a b c)}(x, y) \quad \forall x \in A, y \in B .
$$

Then $S$ is said to be $(A, B)$ generalized cyclic:

(1) $\Lambda$ contraction iff $a+c+b<1$

(2) $\Lambda$ nonexpansive iff $a+c+b=1$

\section{Definition 4}

Let $S: X \rightarrow X$ fulfill the condition:

$$
\begin{aligned}
& d(S(x), S(y)) \leq \Lambda_{S,(a b c)}(x, y) \\
& -\phi\left(\Lambda_{S,(a b c)}(x, y)\right) \quad \forall x \in A, y \in B .
\end{aligned}
$$

where, $\phi$ is lower semi-continuous non-decreasing functions $\phi:[0, \infty] \rightarrow[0, \infty]$ with $\phi(t)>0$ for $t \in[0, \infty]$ and $\phi(0)=0$. Then $S$ is said to be $(A, B)$ generalized cyclic:

(1) $\phi-\Lambda$ weak contraction iff $a+c+b<1$,

(2) $\phi-\Lambda$ weak nonexpansive iff $a+c+b=1$.

\section{Definition 5}

Let $S: X \rightarrow X$ be a mapping fulfill the condition:

$$
\begin{aligned}
& \psi(d(S(x), S(y))) \leq \psi\left(\Lambda_{S,(a b c)}(x, y)\right) \\
& -\phi\left(\Lambda_{S,(a b c)}(x, y)\right) \quad \forall x \in A, y \in B,
\end{aligned}
$$

where, $\psi$ and $\phi$ are lower semi-continuous nondecreasing functions $\psi, \phi:[0, \infty] \rightarrow[0, \infty]$ with $\psi(t)>0$ for $t \in[0, \infty]$ and $\phi(0)=0$ with $\phi(t)>0$ for $t \in[0, \infty]$ and $\phi(0)=0$. Then $S$ is said to be $(A, B)$ generalized cyclic:

(1) $\psi-\phi-\Lambda$ weak contraction iff $a+c+b<1$,

(2) $\psi-\phi-\Lambda$ weak nonexpansive iff $a+c+b=1$.

\section{Definition 6}

Let $S: X \rightarrow X$ be a mapping fulfill the condition:

$$
\begin{aligned}
& \psi(d(S(x), S(y))) \\
& \leq F\left(\psi\left(\Lambda_{S,(a b c)}(x, y)\right), \phi\left(\Lambda_{S,(a b c)}(x, y)\right)\right) \forall x \in A, y \in B,
\end{aligned}
$$

where, $\psi$ and $\phi$ are lower semi-continuous nondecreasing functions $\psi, \phi:[0, \infty] \rightarrow[0, \infty]$ with $\psi(t)>0$ for $t \in[0, \infty], \psi(0)=0, \phi(t)>0$ for $t \in[0, \infty], \phi(0)=0$ and $F$ is a $C$ class function. Then $S$ is said to be $(A, B)$ generalized cyclic:
(1) $F-\psi-\phi-\Lambda$ weak contraction iff $a+c+b<1$,

(2) $F-\psi-\phi-\Lambda$ weak nonexpansive iff $a+c+b=1$.

\section{Example}

Let $X=[-1,1], A=[-1,0]$, and $B=[0,1]$. Define $S: X \rightarrow X$ as:

$$
S(z)=\left\{\begin{array}{l}
-\frac{z}{3}, \text { if } z \in A \\
-\frac{z}{2}, \text { if } z \in B
\end{array}\right.
$$

It is clear that $S$ is cyclic with respect to the representation $A \cup B$ of $X$. Endow $X$ with the metric $d(x$, $y)=|x-y|$, cosider $\phi(t)=t, \psi(t)=t$, and $F(t, s)=t-\frac{1}{2} s$, then the operator $S$ is generalized cyclic $F-\psi-\phi$ $\Lambda\left(\frac{1}{9}\right)\left(\frac{1}{12}\right)\left(\frac{2}{3}\right)$ weak contraction w.r.t $(A, B)$. In fact, let $x \in A$ and $y \in B$. Then we have:

$$
\begin{aligned}
\Lambda_{S\left(\frac{1}{9}\right)\left(\frac{1}{12}\right)\left(\frac{2}{3}\right)}(x, y)=\frac{1}{9} d(x, y)+\frac{1}{12} d(x, S(x)) \\
+\frac{2}{3} \max \{d(y, S(y)), d(y, S(x))\} \\
=\frac{1}{9}(y-x)+\frac{1}{12}\left|x-\left(-\frac{x}{3}\right)\right|+\frac{2}{3} \max \left\{\left|y-\left(-\frac{y}{2}\right)\right|,\left|y-\left(-\frac{x}{3}\right)\right|\right\} \\
=\frac{1}{9}(y-x)+\frac{1}{12}\left|x-\left(-\frac{x}{3}\right)\right|+\frac{2}{3}\left|y-\left(-\frac{y}{2}\right)\right| \\
=\frac{1}{9}(y-x)+\frac{1}{12}\left|x+\frac{x}{3}\right|+\frac{2}{3}\left|y+\frac{y}{2}\right|=\frac{1}{9}(y-x) \\
-\frac{x}{9}+y=\frac{2}{9}(5 y-x) . \\
\quad d(S(x), S(y))=\left|-\frac{x}{3}-\left(-\frac{y}{2}\right)\right|=\left|\frac{y}{2}-\frac{x}{3}\right|=\frac{y}{2}-\frac{x}{3} \\
=\frac{1}{6}(3 y-2 x)=\frac{2}{9}\left(\frac{9}{4} y-\frac{6}{4} x\right) \\
=\frac{2}{9}\left[(5 y-x)-\frac{1}{2}\left(\frac{11}{2} y-x\right)\right] \leq \frac{2}{9}\left[(5 y-x)-\frac{1}{2}(5 y-x)\right] \\
=F\left(\psi\left(\Lambda_{S,(a b c)}(x, y)\right), \phi\left(\Lambda_{S,(a b c)}(x, y)\right)\right) \forall x \in A, y \in B .
\end{aligned}
$$

\section{Remark}

If $\Lambda_{S,(a b c)}(x, y)=a d(x, y) \quad \forall x, y \in X$, that is if $b=c=0$, then we have the usual contraction or nonexpansive mapping acoording to the value of $a, a<1$ or not. One can see some related fixed point theorems proved in the attached references below.

In the light of the particular cases; $F(u, v)=u-v$ and $\psi=I d$; the identity mapping, we noticed the following: 
(1) The class of all $(A, B)$ generalized cyclic $F-\psi-\phi \Lambda$ weak non-expansive is wider than the class of all $(A$, $B)$ generalized cyclic $F$ - $\psi$ - $\phi$ - $\Lambda$ weak contraction.

(2) The class of all $(A, B)$ generalized cyclic $F-\psi-\phi-\Lambda$ weak nonexpansive is wider than the class of all $(A$, $B)$ generalized cyclic $\psi-\phi$ - $\Lambda$ weak nonexpansive.

(3) The class of all $(A, B)$ generalized cyclic $F-\psi-\phi-\Lambda$ weak contraction is wider than the class of all $(A, B)$ generalized cyclic $\psi-\phi$ - $\Lambda$ weak contraction.

(4) The class of all $(A, B)$ generalized cyclic $\psi-\phi-\Lambda$ weak nonexpansive is wider than the class of all $(A, B)$ generalized cyclic $\psi$ - $\phi$ - $\Lambda$ weak contraction.

(5) The class of all $(A, B)$ generalized cyclic $\psi-\phi$ - $\Lambda$ weak nonexpansive is wider than the class of all $(A, B)$ generalized cyclic $\phi$ - $\Lambda$ weak nonexpansive.

(6) The class of all $(A, B)$ generalized cyclic $\phi$ - $\Lambda$ weak nonexpansive is wider than the class of all $(A, B)$ generalized cyclic $\phi-\Lambda$ contraction.

(7) The class of all $(A, B)$ generalized cyclic $\Lambda$ nonexpansive is wider than the class of all $(A, B)$ generalized cyclic $\phi$ - $\Lambda$ weak nonexpansive.

(8) The class of all $(A, B)$ generalized cyclic $\Lambda$ nonexpansive is wider than the class of all $(A, B)$ generalized cyclic $\Lambda$ contraction.

(9) The class of all $(A, B)$ generalized cyclic $\Lambda$ nonexpansive is wider than the class of all $\{a, b, c\}-$ ntype mappings.

(10) The class of all $\{a, b, c\}$-ntype mappings is wider than the class of all $\{a, b, c\}$-ctype mappings.

In this study, the real valued function $\Lambda_{S, T,(a b c)}: X \times$ $X \rightarrow R^{+}$is defined as:

$$
\begin{aligned}
& \Lambda_{S, T(a b c)}(x, y)=a d(x, y)+b d(x, S(x)) \\
& +c \max \{d(y, T(y)), d(y, S(x))\},
\end{aligned}
$$

where, $S, T: X \rightarrow X$ are two self mappings and $a, b, c$ are three real numbers.

We introduced the following fascinating definition for joint-cyclic mapping:

\section{Definition 7}

Let $(X, d)$ be a metric space with $A \cup B, S, T: X \rightarrow X$ be two self mappings and $a, b, c \in[0,1]$ be three real numbers satisfying:

(1) The cyclic condition: $S(A) \subset B$ and $T(B) \subset A$

(2) The contractivity condition:

$$
\begin{aligned}
& d(S(x), T(y)) \leq \Lambda_{S, T,(a b c)}(x, y) \\
& -\phi\left(\Lambda_{S, T,(a b c)}(x, y)\right) \quad \forall x \in A, y \in B,
\end{aligned}
$$

where, $\phi$ is lower semi-continuous non-decreasing function $\phi:[0, \infty] \rightarrow[0, \infty]$ with $\phi(t)>0$ for $t \in[0, \infty]$ and $\phi(0)=0$.

Then $S$ and $T$ are said to be joint $(A, B)$ generalized cyclic:

(1) $\phi$ - $\Lambda$ weak contraction types iff $a+c+b<1$

(2) $\phi$ - $\Lambda$ weak nonexpansive types iff $a+c+b=1$

\section{Definition 8}

Let $(X, d)$ be a metric space with $X=A \cup B, S, T$ : $X \rightarrow X$ be two self mappings and $a, b, c \in[0,1], b \neq 0$ be three real numbers satisfying:

(1) The cyclic condition: $S(A) \subset B$ and $T(B) \subset A$

(2) The contractivity condition:

$$
\begin{aligned}
& \psi(d(S(x), T(y))) \leq \psi\left(\Lambda_{S, T,(a b c)}(x, y)\right) \\
& -\phi\left(\Lambda_{S, T,(a b c)}(x, y)\right) \forall x \in A, y \in B,
\end{aligned}
$$

where, $\psi$ and $\phi$ are non-decreasing functions $\psi, \phi$ : $[0, \infty] \rightarrow[0, \infty]$ with $\psi(t)>0, \phi(t)>0$ for $t \in[0,1]$ and $\phi(0)=0, \phi(0)=0, \psi$ is continuous and $\phi$ is lower semi-continuous.

Then $S$ and $T$ are said to be joint $(A, B)$ cyclic generalized:

(1) $\psi-\phi-\Lambda$ weak contraction types iff $a+c+b<1$

(2) $\psi-\phi-\Lambda$ weak nonexpansive types iff $a+c+b=1$

\section{Definition 9}

Let $(X, d)$ be a metric space with $X=A \cup B, S, T$ : $X \rightarrow X$ be two self mappings and $a, b, c \in[0,1] b \neq 0$ be three real numbers satisfying:

(1) The cyclic condition: $S(A) \subset B$ and $T(B) \subset A$

(2) The following contractivity condition:

$$
\begin{aligned}
& \psi(d(S(x), T(y))) \\
& \leq F\left(\psi\left(\Lambda_{S, T,(a b c)}(x, y)\right), \phi\left(\Lambda_{S, T,(a b c)}(x, y)\right)\right) \forall x \in A, y \in B,
\end{aligned}
$$

where, $\psi$ and $\phi$ are non-decreasing functions $\psi, \phi$ : $[0, \infty] \rightarrow[0, \infty]$ with $\psi(t)>0, \phi(t)>0$ for $t \in[0, \infty]$ and $\psi(0)=0, \phi(0)=0, \psi$ is continuous, $\phi$ is lower semi-continuous and $F$ is some $C$ class function. Then $S$ and $T$ are said to be joint $(A, B)$ generalized cyclic: 
(1) $F-\psi-\phi-\Lambda$ weak contraction types iff $a+c+b<1$

(2) $F-\psi-\phi-\Lambda$ weak nonexpansive types iff $a+c+b=1$

We have the following:

\section{Remarks}

(1) The class of all joint $(A, B)$ generalized cyclic $F-\psi$ $\phi-\Lambda$ weak nonexpansive types is wider than that of joint $(A, B)$ generalized cyclic $\psi-\phi-\Lambda$ weak nonexpansive types.

(2) The class of all joint $(A, B)$ generalized cyclic $F-\psi$ $\phi$ - $\Lambda$ weak nonexpansive types is wider than that of joint $(A, B)$ generalized cyclic $F-\psi-\phi-\Lambda$ weak contraction types.

(3) The class of all joint $(A, B)$ generalized cyclic $\psi-\phi$ $\Lambda$ weak nonexpansive types is wider than that of joint $(A, B)$ generalized cyclic $\psi-\phi-\Lambda$ weak nonexpansive types.

(4) The class of all joint $(A, B)$ generalized cyclic $\psi-\phi$ $\Lambda$ weak nonexpansive types is wider than that of joint $(A, B)$ generalized cyclic $\psi-\phi-\Lambda$ weak contraction types.

(5) If $S, T$ are continuous self mappings on $(X, d)$, then restriction of the mapping $\Lambda_{S, T,(a b c)}: A \times B \rightarrow \mathbb{R}^{+}$for every $x \in A, y \in B$ :

$$
\begin{aligned}
& \Lambda_{S, T,(a b c)}(x, y)=a d(x, y) \\
& +b d(x, S(x))+c \max \{d(y, T(y)), d(y, S(x))\}
\end{aligned}
$$

is continuous.

(6) If $A, B$ are two compact subsets of the metric space $(X, d)$ and $S, T$ are continuous self mappings on $X$, then the restriction of the mapping $\Lambda_{S, T,(a b c)}: A \times B$ $\rightarrow \mathbb{R}^{+}$attains its infimum as well as its supremum at some points in $A \times B$

This paper shows that if $S$ and $T$ are two joint generalized cyclic $F-\psi-\phi-\Lambda$ weak nonexpansive types mappings, then they have only one common fixed point. In particular, every cyclic $C$ class generalized $\psi-\phi-\Lambda$ weak nonexpansive mapping has a unique fixed point. The existing functions $F, \psi$ and $\phi$ give extensions of many results of the references attached in this study.

\section{Main Results}

We have:

\section{Theorem 1}

Let $(X, d)$ be metric space and $A, B$ be two compact subsets of which $X=A \cup B$. If $S, T: X \rightarrow X$ are continuous joint $(A, B)$ generalized cyclic $F-\psi-\phi-\Lambda$ weak nonexpansive mappings on $X$, then there is only one point $z \in X$ such that $S(z)=z=T(z) \in A \cap B$.

\section{Proof}

Let $v_{0}$ be arbitrarily chosen element in $X$. Then $v_{0}$ is either in $A$ or in $B$, if $v_{0}$ is in $B$, then $v_{1}=T\left(v_{0}\right) \in A, v_{2}=$ $S\left(v_{1}\right) \in B, v_{3}=T\left(v_{2}\right) \in A$ and then define by induction:

$v_{2 n+2}=S\left(v_{2 n+1}\right) \in B$ and $v_{2 n+1}=T\left(v_{2 n}\right) \in A \quad \forall n \geq 0$.

First, suppose $n$ is an odd natural number. Then:

$$
\begin{aligned}
& \psi\left(d\left(v_{n+1}, v_{n}\right)\right)=\psi\left(d\left(S\left(v_{n}\right), T\left(v_{n-1}\right)\right)\right) \\
& \leq F\left(\psi\left(\Lambda_{S, T,(a b c)}\left(v_{n}, v_{n-1}\right)\right), \phi\left(\Lambda_{S, T,(a b c)}\left(v_{n}, v_{n-1}\right)\right)\right. \\
& \leq \psi\left(\Lambda_{S, T,(a b c)}\left(v_{n}, v_{n-1}\right)\right) .
\end{aligned}
$$

Since $\psi$ is non-decreasing, we see that:

$$
\begin{aligned}
& d\left(v_{n+1} v_{n}\right) \leq \Lambda_{S, T,(a b c)}\left(v_{n}, v_{n-1}\right) \\
& =a d\left(v_{n}, v_{n-1}\right)+b d\left(S\left(v_{n-1}\right), v_{n-1}\right) \\
& +c \max \left\{d\left(T\left(v_{n}\right), v_{n}\right), d\left(S\left(v_{n-1}\right), v_{n}\right)\right\} \\
& =a d\left(v_{n}, v_{n-1}\right)+b d\left(S\left(v_{n-1}\right), v_{n-1}\right) \\
& +c \max \left\{d\left(v_{n+1}, v_{n}\right), d\left(v_{n}, v_{n}\right)\right\} \\
& =a d\left(v_{n}, v_{n-1}\right)+b d\left(v_{n}, v_{n-1}\right)+c d\left(v_{n+1}, v_{n}\right) \\
& =(a+b) d\left(v_{n}, v_{n-1}\right)+c d\left(v_{n+1}, v_{n}\right) .
\end{aligned}
$$

Thus:

$$
d\left(v_{n+1}, v_{n}\right) \leq\left[\frac{a+c}{1-b}\right] d\left(v_{n}, v_{n-1}\right)=d\left(v_{n}, v_{n-1}\right) .
$$

Therefore:

$$
\begin{aligned}
& \Lambda_{S, T,(a b c)}\left(v_{n}, v_{n-1}\right) \leq(a+b) d\left(v_{n}, v_{n-1}\right)+c d\left(v_{n+1}, v_{n}\right) \\
& \leq(a+b) d\left(v_{n}, v_{n-1}\right)+c d\left(v_{n}, v_{n-1}\right) \\
& =(a+b+c) d\left(v_{n}, v_{n-1}\right)=d\left(v_{n}, v_{n-1}\right) .
\end{aligned}
$$

hence:

$$
d\left(v_{n+1}, v_{n}\right) \leq \Lambda_{S, T,(a b c)}\left(v_{n}, v_{n-1}\right) \leq d\left(v_{n}, v_{n-1}\right) .
$$

Continuing gives:

$$
\begin{aligned}
& d\left(v_{n+1}, v_{n}\right) \leq \Lambda_{S, T,(a b c)}\left(v_{n}, v_{n-1}\right) \\
& \leq d\left(v_{n}, v_{n-1}\right) \leq \Lambda_{S, T,(a b c)}\left(v_{n-1}, v_{n-2}\right)
\end{aligned} .
$$


Second; by a similar method when $n$ is an even natural number, we obtain the same conclusion as inequalities (2.4). Hence the sequences $\left\{d\left(v_{n+1}, v_{n}\right)\right\}_{n \in N}$ and $\left\{\Lambda_{S, T,(a b c)}\left(v_{n+1}, v_{n}\right)\right\}_{n \in N}$ are monotonic non-increasing and bounded below by 0 , thus their limit exist, each equals its infimum.

On the other side; they have the same infimum because of the inequalities (2.4), therefore if their infimum is $r$, then:

$$
\lim _{n \rightarrow \infty} d\left(v_{n+1}, v_{n}\right)=\lim _{n \rightarrow \infty} \Lambda_{S, T,(a b c)}\left(v_{n}, v_{n-1}\right)=r .
$$

Using the properties of $\phi$ :

$$
\phi(r) \leq \liminf _{n \rightarrow \infty} \phi\left(\Lambda_{s, T,(a b c)}\left(v_{n}, v_{n-1}\right)\right) .
$$

Taking least upper limits on two sides of the inequality (2.2) as $n \rightarrow \infty$ gives:

$$
\psi(r) \leq F\left(\psi(r), \liminf _{n \rightarrow \infty} \phi\left(\Lambda_{S, T,(a b c)}\left(v_{n}, v_{n-1}\right)\right) \leq \psi(r) .\right.
$$

Thus:

$$
F\left(\psi(r), \liminf _{n \rightarrow \infty} \phi\left(\Lambda_{S, T,(a b c)}\left(v_{n}, v_{n-1}\right)\right)=\psi(r) .\right.
$$

This insures that either $\psi(r)=0$ or $\lim \inf _{n \rightarrow \infty}$ $\phi\left(\Lambda_{S, T,(a b c)}\left(v_{n}, v_{n-1}\right)=0\right.$. If $\psi(r)=0$, then $r=0$ and if lim $\inf _{n \rightarrow \infty} \phi\left(\Lambda_{S, T,(a b c)}\left(v_{n}, v_{n-1}\right)=0\right.$ while $r>0$, then we have the following contradiction:

$$
0<\phi(r) \leq \liminf _{n \rightarrow \infty} \phi\left(\Lambda_{S, T,(a b c)}\left(v_{n}, v_{n-1}\right)\right)=0 .
$$

Hence:

$$
\lim _{n \rightarrow \infty} d\left(v_{n+1}, v_{n}\right)=\lim _{n \rightarrow \infty} \Lambda_{S, T,(a b c)}\left(v_{n}, v_{n-1}\right)=0 .
$$

This insures that:

$$
\inf \left\{\Lambda_{s, T,(a b c)}(x, y): x \in A, y \in B\right\}=0 .
$$

Since $\Lambda_{S, T,(a b c)}$ attains its infimum on $A \times B$, there is $x_{0} \in A$ and $y_{0} \in B$ such that:

$$
\Lambda_{S, T,(a b c)}\left(x_{0}, y_{0}\right)=0 .
$$

This gives:

$$
\begin{aligned}
& a d\left(x_{0}, y_{0}\right)+b d\left(x_{0}, S\left(x_{0}\right)\right) \\
& +c \max \left\{d\left(y_{0}, T\left(y_{0}\right)\right), d\left(y_{0}, S\left(x_{0}\right)\right)\right\}=0 .
\end{aligned}
$$

Since all are nonnegative real numbers, clearly:

$$
d\left(x_{0}, y_{0}\right)=d\left(x_{0}, S\left(x_{0}\right)\right)=d\left(y_{0}, T\left(y_{0}\right)\right)=d\left(y_{0}, S\left(x_{0}\right)\right)=0,
$$

and we have:

$$
x_{0}=y_{0}=S\left(x_{0}\right)=S\left(y_{0}\right) .
$$

Notice that the converse is also true, if $x_{0}=y_{0}=S\left(x_{0}\right)$ $=T\left(x_{0}\right)=S\left(y_{0}\right)=T\left(y_{0}\right)$, then $\Lambda_{S, T,(a b c)}\left(x_{0}, y_{0}\right)=0$ is clear. On the other side, this showed that $x_{0}=y_{0} \in A \cap B$. If there exists another point $v \in A \cap B$ such that $S(v)=v=T(v)$ with $v \neq y_{0}$, then we get:

$$
\begin{aligned}
& \psi\left(d\left(v, y_{0}\right)\right)=\psi\left(d\left(S(v), T\left(y_{0}\right)\right)\right) \\
& \leq F\left(\psi\left(\Lambda_{S, T,(a b c)}\left(v, y_{0}\right)\right), \phi\left(\Lambda_{S, T,(a b c)}\left(v, y_{0}\right)\right)\right) . \\
& \leq \psi\left(\Lambda_{S, T,(a b c)}\left(v, y_{0}\right)\right) .
\end{aligned}
$$

Hence; the following is a contradiction:

$$
\begin{aligned}
& d\left(v, y_{0}\right) \leq \Lambda_{S, T,(a b c)}\left(v, y_{0}\right) \\
& \leq a d\left(v, y_{0}\right)+b d(S(v), v)+c \max \left\{d\left(y_{0}, T\left(y_{0}\right)\right), d\left(y_{0}, S(v)\right)\right\} \\
& =a d\left(v, y_{0}\right)+c d\left(v, y_{0}\right)=(a+c) d\left(v, y_{0}\right)<d\left(v, y_{0}\right)
\end{aligned}
$$

This shows that $d\left(v, y_{0}\right)=0$, that is $v=y_{0}$.

We have:

\section{Proposition 1}

The sequence defined iteratively by the induction (2.1) is convergent to the unique common fixed point of $S$ and T:

$$
\lim _{n \rightarrow \infty} v_{n}=v .
$$

\section{Proof}

Let $v$ be the unique common fixed point of $S$ and $T$, in addition suppose that $\lim _{n \rightarrow \infty} v_{n}=u$ with $v \neq u$. Then there is $n \in N$ such that:

$$
\begin{aligned}
& \psi\left(d\left(v_{n}, v\right)\right)=\psi\left(d\left(S\left(v_{n-1}\right), T(v)\right)\right) \\
& \leq F\left(\psi\left(\Lambda_{S, T,(a b c)}\left(v_{n-1}, v\right)\right), \phi\left(\Lambda_{S, T,(a b c)}\left(v_{n-1}, v\right)\right)\right. \\
& \leq \psi\left(\Lambda_{S, T,(a b c)}\left(v_{n-1}, v\right)\right) .
\end{aligned}
$$

Hence: 


$$
\begin{aligned}
& \left.d\left(v_{n}, v\right)=d\left(S\left(v_{n-1}\right), T(v)\right)\right) \leq \Lambda_{S, T,(a b c)}\left(v_{n-1}, v\right) \\
& \leq a d\left(v_{n-1}, v\right)+b d\left(S\left(v_{n-1}\right), v_{n-1}\right) \\
& +c \max \left\{d(T(v), v), d\left(S\left(v_{n-1}\right), v\right)\right\} \\
& \leq a d\left(v_{n-1}, v\right)+b d\left(v_{n}, v_{n-1}\right)+c \max \left\{d(v, v), d\left(v_{n}, v\right)\right\} \\
& \leq a d\left(v_{n-1}, v\right)+b d\left(v_{n}, v_{n-1}\right)+c d\left(v_{n}, v\right) .
\end{aligned}
$$

That is:

$$
d\left(v_{n}, v\right) \leq \frac{1}{1-c}\left[a d\left(v_{n-1}, v\right)+b d\left(v_{n}, v_{n-1}\right)\right] .
$$

Using Equation (2.6) with the limiting approach as $n$ $\rightarrow \infty$ prove that:

$$
d(u, v) \leq \frac{a}{1-c} d(u, v)
$$

hence; $\left(1-\frac{a}{1-c}\right) d(u, v) \leq 0$, since $\neq 1-c$, we get $d(u, v)=0$, that is; $v=u$.

\section{Corollary 1}

Let $(X, d)$ be metric space and $A, B$ two compact subsets of which $X=A \cup B$. If $S: X \rightarrow X$ is continuous $(A, B)$ generalized cyclic $F-\psi-\phi$ - $\Lambda$ weak nonexpansive mapping on $X$, then there is only one point $v \in X$ such that $S(v)=v \in A \cap B$. Moreover; for any $v_{0} \in X$, we have $\lim _{n \rightarrow \infty} S^{n}\left(v_{0}\right)=v$.

\section{Proof}

Using Theorem (1) with $S=T$ completes the prove.

\section{Corollary 2}

Let $(X, d)$ be metric space and $A, B$ two compact subsets of which $X=A \cup B$. If $S: X \rightarrow X$ is continuous $(A$, $B)$ generalized cyclic $\psi-\phi-\Lambda$ weak nonexpansive mapping on $X$, then there is only one point $v \in X$ such that $S(v)=v \in A \cap B$. Moreover; for any $v_{0} \in X$, we have $\lim _{n \rightarrow \infty} S^{n}\left(v_{0}\right)=v$.

\section{Proof}

Using Theorem (1) with $S=T$ and taking $F(t, s)=$ $\psi(t)-\phi(s)$ complete the prove.

\section{Corollary 3}

Let $(X, d)$ be metric space and $A, B$ two compact subsets of which $X=A \cup B$. If $S: X \rightarrow X$ is continuous $(A, B)$ generalized cyclic $\phi$ - $\Lambda$ weak nonexpansive mapping on $X$, then there is only one point $v \in X$ such that $S(v)=v \in A \cap B$. Moreover; for any $v_{0} \in X$, we have $\lim _{n \rightarrow \infty} S^{n}\left(v_{0}\right)=v$.

\section{Proof}

Using Theorem (1) with $S=T$, taking $F(t, s)=\psi(t)$ $\phi(s)$ and $\psi(t)=t \forall t \in[0, \infty]$ complete the prove.

\section{Conclusion}

This paper shows that if $S$ and $T$ are two joint generalized cyclic $F-\psi-\phi-\Lambda$ weak nonexpansive type mappings, then they have only one common fixed point. In particular, every generalized cyclic $C$ class $\psi-\phi-\Lambda$ weak nonexpansive mapping has a unique fixed point. Hence continuing restrictions of $F, \psi$ and $\phi$ to be taken special cases gives extensions of many fixed point in the filed of fixed point theory. In particular, it extends the results of attached references in this study.

\section{Acknowledgement}

Sincere thanks go to the valuable comments of the referees and the editors that give a step forward to the main file of the manuscript.

\section{Competing Interests}

The author has no conflict of interests.

\section{References}

Alber, Y.I. and S. Guerre-Delabriere, 1997. Principle of Weakly Contractive Maps in Hilbert Spaces. In: New Results in Operator Theory and its Applications, Gohberg, I. and Y. Lyubich (Eds.), Operator Theory, Advances and Applications, Birkhauser, Basel, Switzerland.

Banach, S., 1922. Sur les operations dans les ensembles absrtacts et leurs applications. Fun. Math., 3: 133-181. DOI: $10.4064 / \mathrm{fm}-3-1-133-181$

Bilgili, N. and E. Karapinar, 2013. Cyclic contractions via auxiliary functions on $\mathrm{G}$ metric spaces. Fixed Point Theory Applic., 2013: 49-49. DOI: 10.1186/1687-1812-2013-49

Bilgili, N., I.M. Erhan, E. Karapinar and D. Turkoglu, 2014. Cyclic contractions and related fixed point theorems on $\mathrm{G}$ metric spaces. Applied Math. Inf. Sci., 8: 1541-1551. DOI: 10.12785/amis/080407

Browder, F., 1979. Remarks on point theorems of contractive type mappings. Nonlinear Anal., 3: 657-661. DOI: 10.1016/0362-546X(79)90094-4

Chatterjea, S.K., 1972. Fixed point theorems. Comptes Rendus del Academie Bulgare des Sciences, 25: 727-730.

Choudhury, B.S., 2009. Unique fixed point theorem for weak C-contractive mappings. J. Sci. Eng. Technol., 5: 6-13. DOI: 10.3126/kuset.v5i1.2842 
Czerwik, S., 1993. Contraction mapping in b metric spaces. Acta Math. Inform. Univ. Ostraviensis, 1: 5-11.

Dutta, P.N. and B.S. Choudhury, 2008. A generalisation of contraction principle in metric spaces. Fixed Point Theory Applic., 2008: 406368. DOI: $10.1155 / 2008 / 406368$

Edelstein, M., 1962. On fixed and periodic points under contractive mappings. J. Lond. Math. Soc., 37: 74-79. DOI: $10.1112 / \mathrm{jlms} / \mathrm{s} 1-37.1 .74$

El-Shobaky, E.M., Sahar Mohamed Ali Abou Bakr and M.S. Ali, 2007. Generalization of Banach contraction principle in two directions. Int. J. Math. Stat., 3: 112- 115.

Harjani, J., B. Lopez and K. Sadarangani, 2013. Fixed point theorems for cyclic weak contractions in compact metric spaces. J. Nonlinear Sci. Applic., 6: 279-284. DOI: 10.22436/jnsa.006.04.05

Hussain, N., E. Karapinar, S. Sedghi, N. Shobe and S. Firouzian, 2014. Cyclic $\phi$ contractions in uniform spaces and related fixed point results. Abs. Applied Anal., 2014: 976859-976859. DOI: $10.1155 / 2014 / 976859$

Jleli, M., E. Karapinar and B. Samet, 2014. On cyclic $(\psi, \phi)$ contractions in Kaleva-Seikkala's type fuzzy metric spaces. J. Intell. Fuzzy Syst., 27: 2045-2053. DOI: $10.3233 /$ IFS-141170

Karapinar, E. and H.K. Nashine, 2012. Fixed point theorem for cyclic Chatterjea type contractions. J. Applied Math., 2012: 165698-165698. DOI: $10.1155 / 2012 / 165698$

Karapinar, E. and K. Sadarangani, 2011. Fixed point theory for cyclic $(\psi, \phi)$ contraction. Fixed Point Theory Applic., 69: 1-8.

Karapinar, E. and K. Sadarangani, 2012. Corrigendum to "Fixed point theory for cyclic weak $\phi$-contraction" [Appl. Math. Lett. 24 (6) (2011) 822-825]. Applied Math. Lett, 25: 1582-1584.

DOI: $10.1016 /$ j.aml.2011.11.001
Karapinar, E. and V. Rakocevic, 2013. On cyclic generalized weakly $\mathrm{C}$ contractions on partial metric spaces. Abs. Applied Anal., 2013: 831491-831491. DOI: $10.1155 / 2013 / 831491$

Karapinar, E., 2011. Fixed point theory for cyclic weak $\phi$ contraction. Applied Math. Lett., 24: 822-825. DOI: $10.1016 /$ j.aml.2010.12.016

Karapinar, E., M. Jleli and B. Samet, 2012. Fixed point results for almost generalized cyclic $(\psi, \phi)$ weak contractive type mappings with applications. Abs. Applied Anal., 2012: 917831. DOI: $10.1155 / 2012 / 793486$

Karapinar, E., S. Romaguera and K. Tas, 2013. Fixed points for cyclic orbital generalized contractions on complete metric spaces. Cent. Eur. J. Math., 11: 552-560. DOI: 10.2478/s11533-012-0145-0

Kirk, W.A., P.S. Srinivasan and P. Veeramani, 2003. Fixed points for mappings satisfying cyclical contractive conditions. Fixed Point Theory, 4: 79-89.

Rhoades, B.E., 2001. Some theorems on weakly contractive maps. Nonlinear Anal., 47: 2683-2693. DOI: 10.1016/S0362-546X(01)00388-1

Sahar Mohamed Ali Abou Bakr., 2009. On fixed point theorem of $\{a, b, c\}$ generalized nonexpansive mappings in normed spaces. J. Egypt. Math. Society, 17: 1-13.

Sahar Mohamed Ali Abou Bakr., 2013. Fixed point theorems of $\{a, b, c\}$ contraction and non-expansive type mappings in weakly Cauchy normed spaces. Anal. Theory Applic., 29: 280-288.

DOI: 10.4208/ata.2013.v29.n3.8

Sahar Mohamed Ali Abou Bakr and A.H. Ansari, 2017. On fixed point theorem of C class functions - B weak cyclic mappings. J. Math. Stat., 13: 312-318. DOI: $10.3844 /$ jmssp.2017.312.318 